\title{
Thermodynamical properties of equiatomic liquid noble metal alloys by a pseudopotential
}

\section{Aditya M. Vora*}

\begin{abstract}
Humanities and Social Science Department, S. T. B. S. College of Diploma Engineering, Shri Swami Atmanand Sarswati Vidya Sankul, Opp. Kapodra Police Station, Varachha Road, Surat 395006, Gujarat, India
\end{abstract}

"Corresponding author. Tel: (+91) 2832-256424, E-mail: voraam@yahoo.com

Received: 16 March 2012, Revised: 22 July 2012 and Accepted: 26 July 2012

\section{ABSTRACT}

The Gibbs-Bogoliubov (GB) inequality is applied to investigate the thermodynamic properties of some equiatomic noble metal alloys in liquid phase such as $\mathrm{Au}-\mathrm{Cu}, \mathrm{Ag}-\mathrm{Cu}$ and $\mathrm{Ag}-\mathrm{Au}$ using well known pseudopotential formalism. For description of the structure, well known Percus-Yevick (PY) hard sphere model is used as a reference system. With this procedure the thermodynamic properties such as entropy and heat of mixing have been computed. The influence of local field correction functions viz; Hartree (HR), Taylor (TY), Ichimaru-Utsumi (IU), Farid et al. (FD) and Sarkar et al. (SS) is also investigated and found successful. The computed results of the excess entropy and the heats of mixing compares are found in qualitative agreement with the available experimental or theoretical data in the literature. Copyright $@ 2013$ VBRI press.

Keywords: Gibbs-Bogoliubov inequality; equiatomic liquid noble metal alloys; pseudopotential method; Percus-Yevick hard sphere model; local field correction functions; thermodynamic properties.

PACS Nos. 67.55.Cx, 65.40.Gr, 71.15.Dx

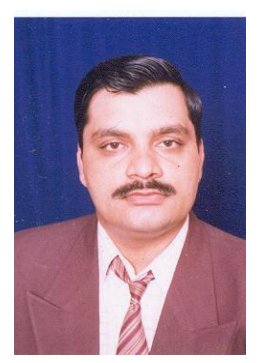

A. M. Vora obtained his Ph. D. in Physics from Sardar Patel University, Vidhyanagar, Anand, Gujarat, India in 2004. Presently, he is working as an I/C Head of Humanities and Social Science Department at STBS College of Diploma Engineering, Surat, India since 2008. His trust area of working is Experimental Condensed Matter as well as Theoretical Condensed Matter and Computational Physics. He involves with in the field of studying physical properties like thermodynamical, structural, transport, surface, magnetic, vibrational properties of liquid metals as well as their alloys and non-crystalline materials using pseudopotential approach.

\section{Introduction}

The theoretical basis for an understanding of the thermodynamics of simple liquid metals has been forged in recent years to a point where it can be used to calculate the thermodynamic properties with some success. This advance has been made possible due to the combination of the pseudopotential with thermodynamic perturbation theories. The pseudopotential theory enables one to formulate the energy in terms of the pseudopotential and of the structure factor [1-21]. This Gibbs-Bogoliubov (GB) inequality states that when the Hamiltonian of a given system is regarded as the Hamiltonian of a reference system plus a perturbation, the free energy of the system will always be smaller than that of the reference system plus the expectation values of the perturbation (calculated with the structure factors of the reference system). In our case, the reference system is one of the hard spheres, and the hard sphere diameters will be chosen to minimize the free energy. Recently, Chekmarev et al. [22] have been reported structural information of alkali metals in liquidvapor interface using a simple model potential. Also, very recently Ghatee and Bahadori [23] have reported Interionic potential function in liquid alkali metals by thermodynamic regularity approach.

Though the theoretical formulation for investigating thermodynamical properties of binary alloys is very straight forward, from the pseudopotential point of view, a few attempts have been found to compute such properties as a function of concentration [1-21]. Hence, we are motivated to compute the thermodynamical properties as a function of concentration like internal energy, entropy, Helmholtz free energy, entropy of mixing and heat of mixing of liquid binary alloys of alkali-alkali component. We have used our well recognized model potential $[\mathbf{2 4}, \mathbf{2 5}]$ in the present computation. The modified Hartree dielectric function [26] together with the exchange and correlation effects proposed by Hartree (HR) [26], Taylor (TY) [27], Ichimaru-Utsumi (IU) [28], Farid et al. (FD) [29] and Sarkar et al. (SS) [30] of the conduction electrons 
generates good energy wave number characteristics. Very recently, various researchers have studied transport and thermodynamical properties of crystalline materials [3133].

\section{Computational methodology}

Our well recognized model potential [24, 25] employed in the present study has the real space form (in atomic units)

$$
\begin{aligned}
W(r) & =-\frac{Z e^{2}}{r_{c}^{3}}\left[2-\exp \left(1-\frac{r}{r_{c}}\right)\right] r^{2} ; \quad r \leq r_{c} \\
& =-\frac{Z e^{2}}{r} \\
& ; \quad r \geq r_{c}
\end{aligned}
$$

This form has a feature of the Columbic term outside the core and varying cancellation due to a repulsive and an attractive contribution to the potential within the core. Hence it is assumed that the potential within the core should not be zero nor constant but it should very as a function of $r$. Thus the model potential has the novel feature of representing varying cancellation of potential within the core over and above its continuity and weak nature. The wave number representation of the model potential form factor (in atomic units) is $[\mathbf{2 4}, \mathbf{2 5}]$.

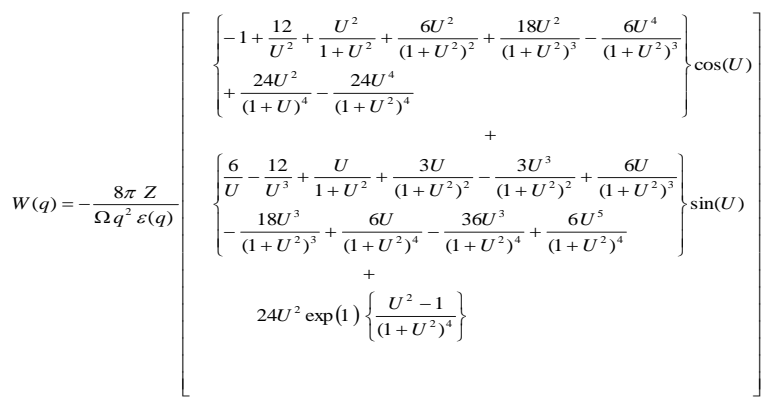

Here $U=q r_{C}$. The parameters $Z, e, \Omega_{O}, q, r_{C}$ and $\varepsilon(q)$ are the valence, charge of the electron, atomic volume, wave vector, parameter of the model potential and modified dielectric function, respectively. The parameter of the model potential and modified dielectric function, respectively. The parameter of the model potential $r_{C}$ is determined using the first zero of the form factor [24, 25].

We consider an alloy having $(1-x) N$ atoms of type 1 at positions $\left\{R_{1}\right\}$ and $x N$ atoms of type 2 at positions $\left\{R_{2}\right\}$. Also consider $(1-x)+x=1$ so that $N$ is the total number of atoms. The number densities of the ion species are $n_{1}=(1-x) n$ and $n_{2}=x n$, here $n=N / \Omega_{O}$ with $\Omega_{O}$ is the total volume of the alloy. If $Z_{1}$ and $Z_{2}$ are the valences, then the free electron Fermi wave vector is given by $k_{F}^{3}=3 \pi^{2} \bar{Z} n$, where $\bar{Z} n=Z_{1} n_{1}+Z_{2} n_{2}$, the mean average valence electron density [6].
The electronic free energy of the alloy for some fixed configuration is obtained by [6]

$F_{e l}\left\{R_{1}\right\},\left\{R_{2}\right\}=F_{e g}+F_{1}+F_{2}\left(\left\{R_{1}\right\},\left\{R_{2}\right\}\right)$.

Where, $F_{e g}$ is the free energy of the electron gas, $F_{1}$ and $F_{2}$ are obtained via first and second order pseudopotential theory.

By adding direct Coulomb interaction between ions, one obtains the effective potential energy for the ion system. We need only the expectation value of this effective potential averaged over some reference system, which is given by [6],

$F_{p s}=F_{e g}+F_{M}+F_{1}+F_{2}$.

$F_{e g}$ is given by [6],

$F_{e g}=\left[\frac{3}{10} k_{F}^{3}-\frac{3}{4 \pi} k_{F}+E_{\text {core }}-\frac{1}{2} \gamma_{e g} T^{2}\right] \bar{Z}$,

here $\quad E_{\text {core }}=-0.0474-0.0155 \ln k_{F} \quad$ is the correlation energy contribution. The constant $\gamma_{e g}=\left(\pi k_{B} / k_{F}\right)^{2}$ is the low temperature specific heat of the electron gas.

The Madelung contribution (FM) can be written as [6],

$F_{M}=c_{1}^{2} Z_{1}^{2} I_{11}+c_{2}^{2} Z_{2}^{2} I_{22}+2 c_{1} c_{2} Z_{1} Z_{2} I_{12}$

where,

$I_{11}=[\pi n /(1+2 \eta)]\left[-\sigma_{1}^{2}+\frac{1}{5}(1-\eta / 2)\left(\eta_{1} \sigma_{1}^{2}+\eta_{2} \sigma_{2}^{2}\right)+\eta_{1} \sigma_{1}\left(\sigma_{2}-\sigma_{1}\right)+\frac{1}{2} \eta_{1} \eta_{2}\left(\sigma_{2}-\sigma_{1}\right)^{2}\right]$,

$I_{12}=[\pi n /(1+2 \eta)]\left[\begin{array}{l}-\frac{1}{4}\left(\sigma_{1}+\sigma_{2}\right)^{2}+\frac{1}{5}(1-\eta / 2)\left(\eta_{1} \sigma_{1}^{2}+\eta_{2} \sigma_{2}^{2}\right)+\frac{1}{2}\left(\eta_{2} \sigma_{1}-\eta_{1} \sigma_{2}\right)\left(\sigma_{2}-\sigma_{1}\right) \\ +\frac{1}{2} \eta_{1} \eta_{2}\left(\sigma_{2}-\sigma_{1}\right)^{2}\end{array}\right]$,

The expressions for $I_{22}$ is obtained by exchanging $\eta_{1}, \sigma_{1}$ with $\eta_{2}, \sigma_{2}$ in the expression for $I_{11}$. The temperature dependent packing fraction $\eta$ of the equiatomic liquid noble metal alloys is expressed as [34].

$\eta=A_{W} \exp \left(-B_{W} T\right)$.

Where $A_{W}, B_{W}$ and $T$ are the Waseda's [34] parameters and the absolute temperature of the metallic complexes, respectively. From the values of the packing 
fraction $\eta$, the hard sphere diameter $\sigma$ can be calculated from $\sigma=(6 \eta / \pi \rho)^{1 / 3}$ with $\rho$ is the number density.

Using zeroth Fourier component of the bare pseudopotential $F_{1}$ is obtained. If we denote the $q^{\text {th }}$ components by $W_{B i}(q),(i=1,2)$, then [6].

$F_{1}=\left(c_{1} \alpha_{1}+c_{2} \alpha_{2}\right) \bar{Z} n$.

(10)

where,

$\alpha_{i}=\lim _{q \rightarrow 0}\left[W_{B i}(q)+\frac{4 \pi Z_{i} e^{2}}{q^{2}}\right]$

The second order or band structure energy $F_{2}\left(\left\{R_{1}\right\},\left\{R_{2}\right\}\right)$ is given by

$F_{2}=\frac{1}{16 \pi^{3}} \int_{0}^{\infty}\left\{\begin{array}{l}c_{1} c_{2}\left(W_{B 1}-W_{B 2}\right)^{2}+c_{1}^{2} W_{B 1}^{2} a_{11} \\ +2\left(c_{1} c_{2}\right) \frac{1}{2} W_{B 1} W_{B 2}+c_{2}^{2} W_{B 2}^{2} a_{22}\end{array}\right\}\left(\frac{1}{\varepsilon(q)}-1\right) d q-\frac{\bar{Z}}{2} \gamma_{2}(T) T^{2}$

The $\varepsilon(q)$ the modified Hartree (HR) screening function [26] which is given by

$\varepsilon(\mathrm{q})=1+\left(\varepsilon_{\mathrm{H}}(\mathrm{y})-1\right)(1-\mathrm{G}(\mathrm{y})) ; y=\frac{q}{2 k_{F}}$,

here $\varepsilon_{\mathrm{H}}(\mathrm{y})$ is the static Hartree (HR) screening function [26] given by,

$\varepsilon_{H}(y)=1+\frac{\mathrm{me}^{2}}{2 \pi k_{F} \hbar^{2} \eta^{2}}\left(\frac{1-\mathrm{y}^{2}}{2 \mathrm{y}} \ln \left|\frac{1+\mathrm{y}}{1-\mathrm{y}}\right|+1\right) ; y=\frac{q}{2 k_{F}}$

While $G(y)$ is the local field correction function. In the present investigation, the local field correction functions due to Hartree (HR) [26], Taylor (TY) [27], Ichimaru-Utsumi (IU) [28], Farid et al. (FD) [29] and Sarkar et al. (SS) [30] are incorporated to see the impact of exchange and correlation effects. The details of the local field corrections are narrated below.

$\gamma_{2}(T)$, the second order correction to the usual $\gamma$ factor describing the low temperature electronic specific heat and is given by [6],

$\gamma_{2}(T)=\frac{2 k_{B}^{2}}{3 \pi^{2} \bar{Z}} \int_{0}^{\infty} d y \frac{y^{2}}{y^{2}-1} f(y)\left\{\begin{array}{l}c_{1} c_{2}\left(W_{B 1}-W_{B 2}\right)^{2}+c_{1}^{2} W_{B 1}^{2} a_{11} \\ +2\left(c_{1} c_{2}\right) \frac{1}{2} W_{B 1} W_{B 2}+c_{2}^{2} W_{B 2}^{2} a_{22}\end{array}\right\}$

with, $f(y)=\frac{1}{2}+\frac{y^{2}-1}{4 y} \ln \left|\frac{1+y}{1-y}\right| ; y=\frac{q}{2 k_{F}}$.

Here, $a_{i i}(\mathrm{i}=1,2)$ denotes the structure factor of liquid alloys computed from the mathematical expression given by Ashcroft and Langreth [36, 37].

The free energy per particle of the mixture can be written as [6],

$$
F_{h s}=c_{1} \mu_{1}+c_{1} \mu_{1}-\frac{P_{h s}}{n},
$$

here $\mu_{i}(i=1,2)$ and $P_{h s}$ are the chemical potentials of the components and the pressure respectively. In PY approximation, such quantities are written by [6],

$\frac{\mu_{i}}{k_{B} T}=\ln \left(n_{i} \frac{2 \pi 2^{2}}{m_{i} k_{B} T}\right)^{\frac{3}{2}}-\ln (1-\eta)+\ln \left(\frac{3 X \sigma_{i}}{1-\eta}\right)+\frac{3}{2}\left(\frac{3 X^{2}}{(1-\eta)^{2}}+\frac{2 Y}{1-\eta}\right) \sigma_{i}^{2}+\left(\frac{\pi P_{h s} \sigma_{i}^{3}}{6 k_{B} T}\right.$,

And

$\frac{P_{h s}}{k_{B} T}=\frac{n\left(1+\eta+\eta^{2}\right)-\frac{1}{2} \pi n_{1} n_{2}\left(\sigma_{1}-\sigma_{2}\right)^{2}\left(\sigma_{1}+\sigma_{2}+\sigma_{1} \sigma_{2} X\right)}{(1-\eta)^{3}}$

where,

$$
X=\frac{1}{6} \pi\left(n_{1} \sigma_{1}^{2}+n_{2} \sigma_{2}^{2}\right)
$$

And

$Y=\frac{1}{6} \pi\left(n_{1} \sigma_{1}+n_{2} \sigma_{2}\right)$.

Here, $m_{i}(i=1,2)$ denote the masses of the spheres.

Substitution of the equations (18-20) in (17) and use of $S=-(\partial F / \partial T)_{\Omega_{O}}$, we get

$$
F_{h s}=\frac{3}{2} k_{B} T-T S_{h s}
$$

where

$$
S_{h s}=S_{g a s}+S_{c}+S_{\eta}+S_{\sigma}
$$

With

$$
\frac{S_{g a s}}{k_{B}}=\ln \left(\frac{e}{n}\left(\frac{e m k_{B} T}{2 \pi \hbar^{2}}\right)^{\frac{3}{2}}\right) ; \quad\left(m=m_{1}^{c_{1}} m_{2}^{c_{2}}\right),
$$




$$
\begin{aligned}
& \frac{S_{c}}{k_{B}}=-\left(c_{1} \ln c_{1}+c_{2} \ln c_{2}\right), \\
& \frac{S_{\eta}}{k_{B}}=\ln (1-\eta)+\frac{3}{2}\left(1-\frac{1}{(1-\eta)^{2}}\right), \\
& \text { and }
\end{aligned}
$$

$$
\frac{S_{\sigma}}{k_{B}}=\frac{\pi c_{1} c_{2} n\left(\sigma_{1}-\sigma_{2}\right)^{2}\left(12\left(\sigma_{1}+\sigma_{2}\right)-\pi n\left(c_{1} \sigma_{1}^{4}+c_{2} \sigma_{2}^{4}\right)^{2}\right)}{24(1-\eta)^{2}} .
$$

The Helmholtz free energy ( $F$ ) per ion of the alloy is given as [6]

$F=F_{p s}+F_{h s}$.

Therefore, the above condition directly gives the free energy, internal energy and entropy (or excess entropy). The heat of mixing is calculated from the equation below $[6,8]$.

$\Delta F_{\text {mixing }}=\Delta F_{\text {eg }}+\Delta F_{1}+\Delta F_{2}+\Delta F_{M}$,

With

$$
\Delta F_{i}=F_{i}(\text { alloy })-C_{1} F_{1 A}-C_{2} F_{2 B} .
$$

Here ' $i$ ' denotes 1,2 and $M$. While $C_{1}=(1-x)$ and $C_{2}=x$ are the concentrations of the two metallic elements. Also, $A$ and $B$ stands for constituent elements. The excess entropy is given by

$$
\Delta S=\Delta S_{g a s}+\Delta S_{\eta}+\Delta S_{\sigma}+\Delta S_{C},
$$

with $\Delta S_{p}=S_{p}($ alloy $)-C_{1} S_{1 A}-C_{2} S_{2 B}$.

Where ' $p$ ' corresponds to gas, $\eta, \sigma$ and $C$ terms.

Table 1. Input parameters and other constants.

\begin{tabular}{ccccc}
\hline Met. Ele. & $Z$ & $\begin{array}{c}M \\
(\mathrm{amu})\end{array}$ & $\begin{array}{c}\Omega_{O} \\
(\mathrm{au})\end{array}$ & $r_{C}(\mathrm{au})$ \\
\hline $\mathrm{Cu}$ & 1 & 63.55 & 89.54 & 1.0299 \\
$\mathrm{Ag}$ & 1 & 107.87 & 130.70 & 1.3450 \\
$\mathrm{Au}$ & 1 & 196.97 & 127.70 & 1.0372 \\
\hline
\end{tabular}

\section{Results and discussion}

All computations are performed for the equiatomic liquid noble metal alloys. The input parameters and constants used in the present investigation of the thermodynamic properties are tabulated in Tables 1-2. The input parameters given in Table $\mathbf{1}$ are taken from the literature $[37,38]$. The effective valance has been taken as one in all the components and all quantities are expressed in atomic units.

The results of the thermodynamic properties of liquid noble metal alloys are shown in Tables 3-7 for equiatomic composition. It is seen from Table 3 that $S_{\eta}$ is negative while the remaining contributions are positive. The largest contribution to the total entropy comes from the term $S_{\text {gas }}$ while the smallest arises from $S_{\eta}$ that depends only on the value of packing density $\eta$. The contribution of $S_{\text {gas }}$ increases the absolute value of the total entropy whereas $S_{\eta}$ tends to decrease the total entropy of the system. As we have already stated, $S_{c}$ is the ideal entropy of mixing, its magnitude remains same for all equiatomic liquid noble metal alloys. All the contributions of the entropy are independent of model potential used in the present investigation as well as not influenced by the nature of the exchange and correlation functions.

Table 2.Calculated packing fractions $(\eta)$.

\begin{tabular}{ccccc}
\hline Metallic & & $\eta_{1}$ & $\eta_{2}$ & $\eta_{12}$ \\
Elements & $T(\mathrm{~K})$ & & - & - \\
$\mathrm{Cu}$ & & 0.46 & -45 & - \\
$\mathrm{Ag}$ & 1423 & - & 0.45 & 0.45 \\
$\mathrm{Cu}-\mathrm{Ag}$ & & 0.46 & 0.45 & - \\
$\mathrm{Ag}$ & & 0.46 & - & - \\
$\mathrm{Au}$ & 1350 & - & 0.44 & - \\
$\mathrm{Ag}-\mathrm{Au}$ & & 0.46 & 0.44 & - \\
$\mathrm{Cu}$ & & 0.44 & - & - \\
$\mathrm{Au}$ & 1550 & - & 0.42 & 0.43 \\
$\mathrm{Au}-\mathrm{Cu}$ & & 0.44 & 0.42 & \\
\hline
\end{tabular}

Also, from Tables 4-6 it is observed that, the values of $F_{e g}, F_{2}$ and $F_{M}$ is negative contribution to the internal energy. The contributions $F_{1}$ and $F_{2}$ are potential dependent while other i.e. $F_{e g}$ and $F_{M}$ are independent of the potential. Here, we have used PY partial structure factor $[35,36]$ to describe the structural behaviour. It is seen that the local field correction functions affect the term $F_{2}$ only, which is the second order band structure energies.

It is noticed that the major contribution to the internal energy comes from the structural part of the energy. The magnitude of the Madelung energy $\left|F_{M}\right|$, is quite large, in comparison with other energy terms. Its magnitude 
decreases with an increase in the atomic fraction of the heavier element. The energy of the electron gas $F_{e g}$, in the mixture varies slightly with respect to temperature of the metallic elements. The negative contribution to the energy comes from the first-order pseudopotential term $F_{1}$, which decreases as we increase the concentration of the heavier element. The modulus of the band structure energy $\left|F_{2}\right|$ has a maximum in the intermediate range of concentration. However, the aggregate effects of different energy terms are such that the total internal energy of the system is found to be almost a linear. And hence, the Helmholtz free energy $(F)$ of the system is also found to be almost a linear nature for most of the equiatomic alloys.

Table 3. Various contributions to the Entropy ( $S$ ) of equiatomic liquid noble metals and their equiatomic alloys (in $1 / \mathrm{k}_{\mathrm{B}}$ units).

\begin{tabular}{|c|c|c|c|c|c|c|}
\hline $\begin{array}{l}\text { Metallic } \\
\text { Elements }\end{array}$ & $\begin{array}{l}\mathbf{T} \\
(\mathbf{K})\end{array}$ & $S_{\text {gas }}$ & $S_{C}$ & $S_{\eta}$ & $S_{\sigma}$ & $S$ \\
\hline $\mathrm{Cu}$ & & 13.6243 & 0 & $-\overline{4.1734}$ & 0 & 9.4510 \\
\hline $\mathrm{Ag}$ & 1423 & 14.7956 & 0 & $-\overline{4.0662}$ & 0 & 10.7294 \\
\hline $\mathrm{Ag}-\mathrm{Cu}$ & & 14.2277 & 0.6931 & - & 0.0295 & 10.8309 \\
\hline $\mathrm{Ag}$ & & 14.7092 & 0 & $-\overline{4.1866}$ & 0 & 10.5226 \\
\hline $\mathrm{Au}$ & 1350 & 15.5970 & 0 & $\begin{array}{l}- \\
3.8383\end{array}$ & 0 & 11.7587 \\
\hline $\mathrm{Ag}-\mathrm{Au}$ & & 15.1531 & 0.6931 & - & 0.0008 & 11.8385 \\
\hline $\mathrm{Cu}$ & & 13.7525 & 0 & $\overline{3.9474}$ & 0 & 9.8052 \\
\hline $\mathrm{Au}$ & 1550 & 15.8042 & 0 & $\begin{array}{l}- \\
3.5249\end{array}$ & 0 & 12.2793 \\
\hline $\mathrm{Au}-\mathrm{Cu}$ & & 14.7940 & 0.6931 & $\begin{array}{l}- \\
3.7301\end{array}$ & 0.0179 & 11.7750 \\
\hline
\end{tabular}

It is seen that the exchange and correlation effects affect the numerical values of Helmholtz free energy $(F)$. It is also found that the influence of local field correction functions is very small. The Helmholtz free energy $(F)$ obtained due to the TY, IU and SS-local field correction functions lies between those obtained due to HR and FDlocal field correction functions, in general. As the volume of a particular system increases, it decreases the magnitude of the internal energy as well as the Helmholtz free energy $(F)$ of the system. The present results of the major contribution to the Helmholtz free energy $(F)$ are found in qualitative agreement with the reported theoretical data [8].

The percentile influence of the various local field correction functions with respect to the static HRscreening function on $F_{2}$ is observed in the range of 13.70\%-28.77\% for $\mathrm{Cu}-\mathrm{Ag}$ alloys, $17.34 \%-36.84 \%$ for $\mathrm{Ag}-$ $\mathrm{Au}$ alloys and $14.29 \%-31.43 \%$ for $\mathrm{Cu}-\mathrm{Ag}$ alloys, respectively. While those influences on $F$ is observed in the range of $0.83 \%-1.74 \%$ for $\mathrm{Cu}-\mathrm{Ag}$ alloys, $0.49 \%-1.02 \%$ for $\mathrm{Ag}-\mathrm{Au}$ alloys and $0.44 \%-1.02 \%$ for $\mathrm{Cu}-\mathrm{Ag}$ alloys, respectively.

In Table 7, the entropy of formation and heat of mixing have been narrated for equiatomic composition of liquid noble metals. The presently computed results of the excess entropy of equiatomic liquid noble metal alloys are found quite satisfactory with the available experimental or theoretical data in the literature [8]. The heat of mixing of equiatomic liquid noble metal alloys is found comparatively a very high energy and values with the available theoretical or experimental yielding [8]. The higher values of the heat of mixing for equiatomic liquid noble metal alloys may be due to the large contribution to it from the first order energy term. This may be because of the assumption of ideal mixing which may not be valid in the alloys. The heat of mixing of the equiatomic Ag-Cu is found in qualitative agreement with other theoretical data [8].

Table 4. Various contributions to the Helmholtz free energy ( $F$ ) (in atomic units) of equiatomic $\mathrm{Cu}-\mathrm{Ag}$ alloy.

\begin{tabular}{|c|c|c|c|c|c|c|c|c|c|c|c|c|c|c|c|c|}
\hline \multirow{2}{*}{$\begin{array}{l}\text { Met. } \\
\text { Ele. }\end{array}$} & \multirow[b]{2}{*}{$\mathrm{T}(\mathrm{K})$} & \multirow[b]{2}{*}{ SCR } & \multicolumn{2}{|c|}{$F_{1}$} & \multicolumn{2}{|c|}{$F_{2}$} & \multicolumn{2}{|c|}{$F_{M}$} & \multicolumn{2}{|c|}{$3 / 2 k_{B} T$} & \multicolumn{2}{|c|}{$F_{h s}$} & \multicolumn{2}{|c|}{$F_{e g}$} & \multicolumn{2}{|c|}{$F$} \\
\hline & & & Present & $\begin{array}{c}\text { Others } \\
{[8]}\end{array}$ & Present & $\begin{array}{c}\text { Others } \\
{[8]}\end{array}$ & Present & $\begin{array}{c}\text { Others } \\
{[8]}\end{array}$ & Present & $\begin{array}{c}\text { Others } \\
{[8]}\end{array}$ & Present & $\begin{array}{c}\text { Others } \\
{[8]}\end{array}$ & Present & $\begin{array}{c}\text { Others } \\
{[8]}\end{array}$ & Present & $\begin{array}{c}\text { Others } \\
{[8]}\end{array}$ \\
\hline \multirow{5}{*}{$\mathrm{Cu}$} & \multirow{15}{*}{1423} & HR & \multirow{5}{*}{0.4324} & \multirow{5}{*}{0.2037} & -0.0097 & \multirow{5}{*}{-0.126} & \multirow{5}{*}{-0.3113} & \multirow{5}{*}{-0.312} & \multirow{15}{*}{0.0068} & \multirow{15}{*}{0.0068} & \multirow{5}{*}{-0.0358} & \multirow{5}{*}{-0.358} & \multirow{5}{*}{-0.063} & \multirow{5}{*}{-0.063} & -0.0638 & \multirow{5}{*}{-0.309} \\
\hline & & TY & & & -0.0070 & & & & & & & & & & -0.0612 & \\
\hline & & $\mathrm{IU}$ & & & -0.0083 & & & & & & & & & & -0.0624 & \\
\hline & & FD & & & -0.0067 & & & & & & & & & & -0.0608 & \\
\hline & & SS & & & -0.0065 & & & & & & & & & & -0.0607 & \\
\hline \multirow{5}{*}{$\mathrm{Ag}$} & & HR & \multirow{5}{*}{0.3472} & \multirow{5}{*}{0.0933} & -0.0076 & \multirow{5}{*}{-0.164} & \multirow{5}{*}{-0.2740} & \multirow{5}{*}{-0.271} & & & \multirow{5}{*}{-0.0416} & \multirow{5}{*}{-0.043} & & & -0.1159 & \\
\hline & & TY & & & -0.0060 & & & & & & & & & & -0.1144 & \\
\hline & & $\mathrm{IU}$ & & & -0.0067 & & & & & & & & -0.074 & -0.074 & -0.1150 & -0.447 \\
\hline & & FD & & & -0.0058 & & & & & & & & & & -0.1142 & \\
\hline & & SS & & & -0.0057 & & & & & & & & & & -0.1141 & \\
\hline & & HR & & & -0.0073 & & & & & & & & & & -0.1206 & \\
\hline & & TY & & & -0.0055 & & & & & & & & & & -0.1188 & \\
\hline $\mathrm{Cu}-\mathrm{Ag}$ & & $\mathrm{IU}$ & 0.3818 & 0.1385 & -0.0063 & -0.152 & -0.2832 & -0.290 & & & -0.0421 & -0.046 & -0.070 & -0.070 & -0.1196 & -0.403 \\
\hline & & FD & & & -0.0052 & & & & & & & & & & -0.1186 & \\
\hline & & SS & & & -0.0052 & & & & & & & & & & -0.1185 & \\
\hline
\end{tabular}


Table 5. Various contributions to the Helmholtz free energy ( $F$ ) (in atomic units) of equiatomic Ag-Au alloy.

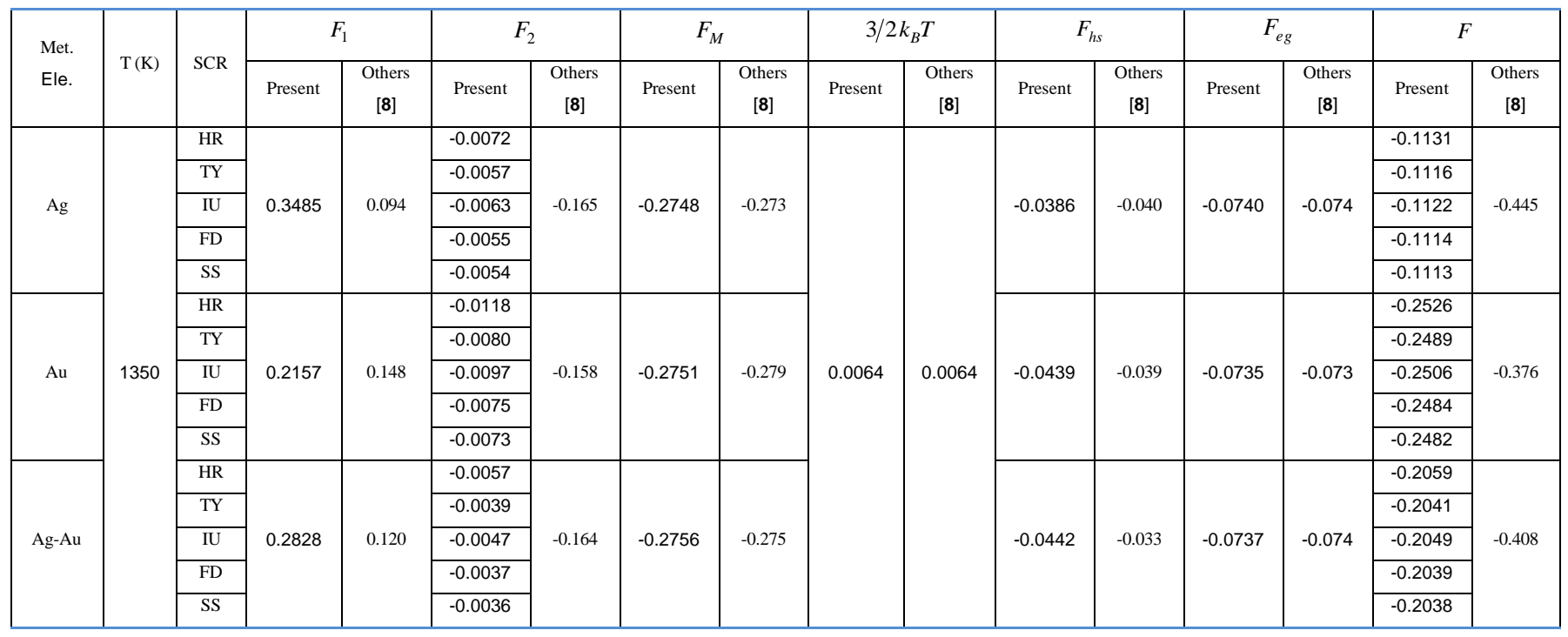

Table 6. Various contributions to the Helmholtz free energy ( $F$ ) (in atomic units) of equiatomic Cu-Au alloy.

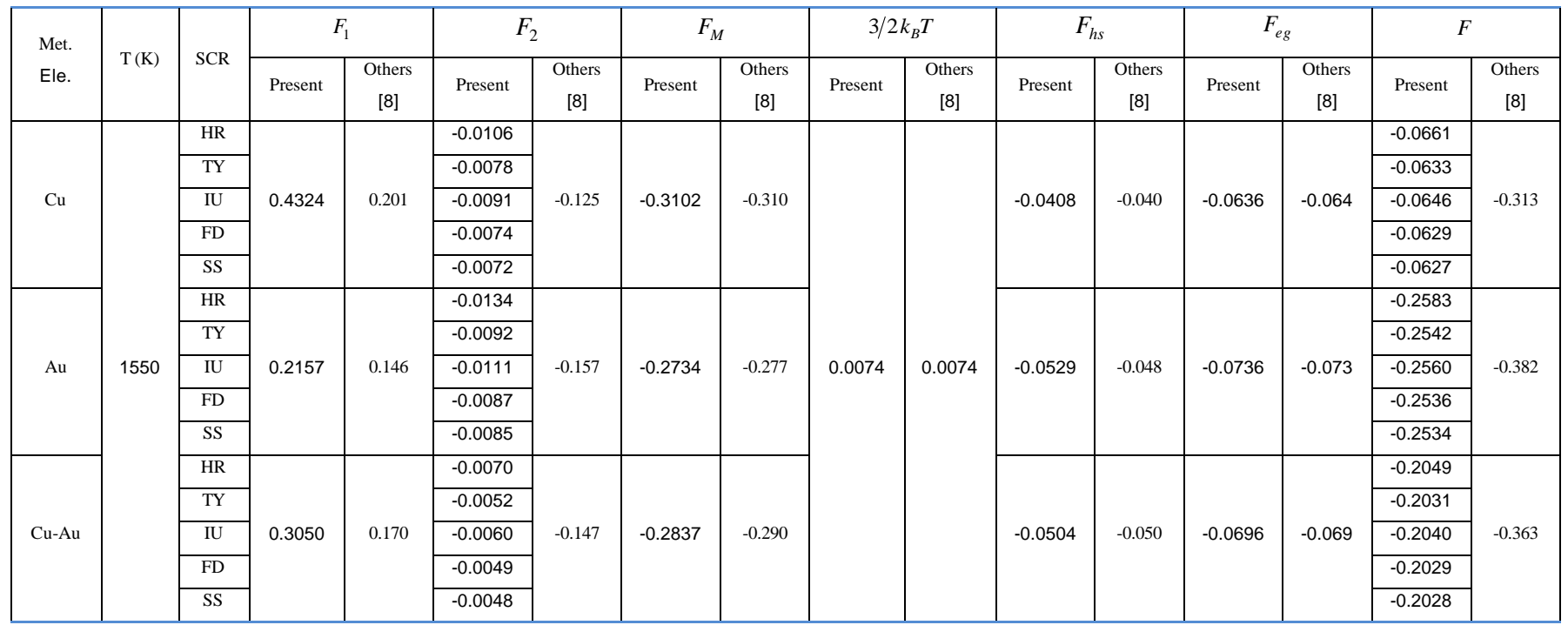

Table 7. Excess entropies and heat of mixing of equiatomic liquid noble metal alloys.

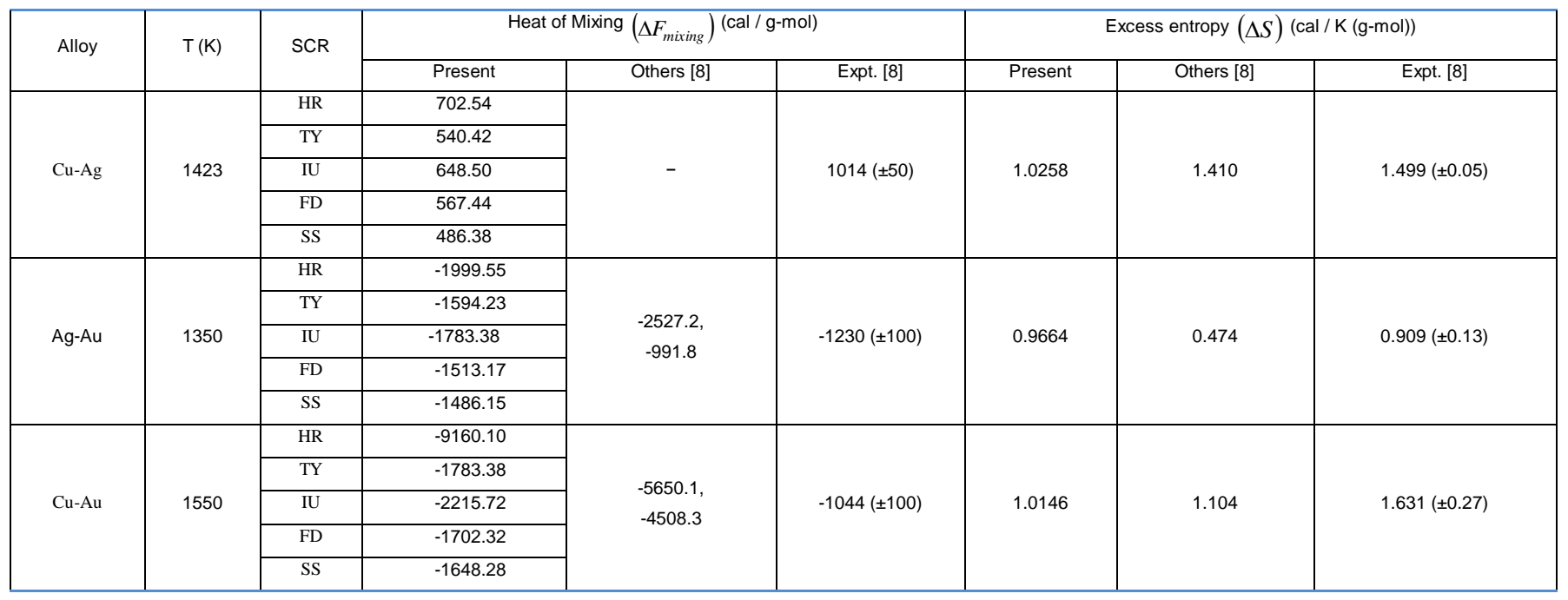


The percentile influence of the various local field correction functions with respect to the static HRscreening function on heat of mixing is observed in the range of $7.69 \%-30.77 \%$ for $\mathrm{Cu}-\mathrm{Ag}$ alloys, $10.81 \%-25.68 \%$ for $\mathrm{Ag}-\mathrm{Au}$ alloys and $75.81 \%-82.01 \%$ for $\mathrm{Cu}-\mathrm{Ag}$ alloys, respectively. The calculated results of the heat of mixing for $\mathrm{Cu}-\mathrm{Ag}, \mathrm{Ag}-\mathrm{Au}$ and $\mathrm{Cu}-\mathrm{Ag}$ alloys deviate in the range of $30.72 \%-72.03 \%, 20.83 \%-62.57 \%$ and $63.06 \%-777.40 \%$ from the experimental findings, respectively. While presently computed results of the excess entropy for $\mathrm{Cu}$ $\mathrm{Ag}, \mathrm{Ag}-\mathrm{Au}$ and $\mathrm{Cu}-\mathrm{Ag}$ alloys deviate in the range of $31.57 \%, 6.31 \%$ and $37.79 \%$ from the experimental findings [8], respectively.

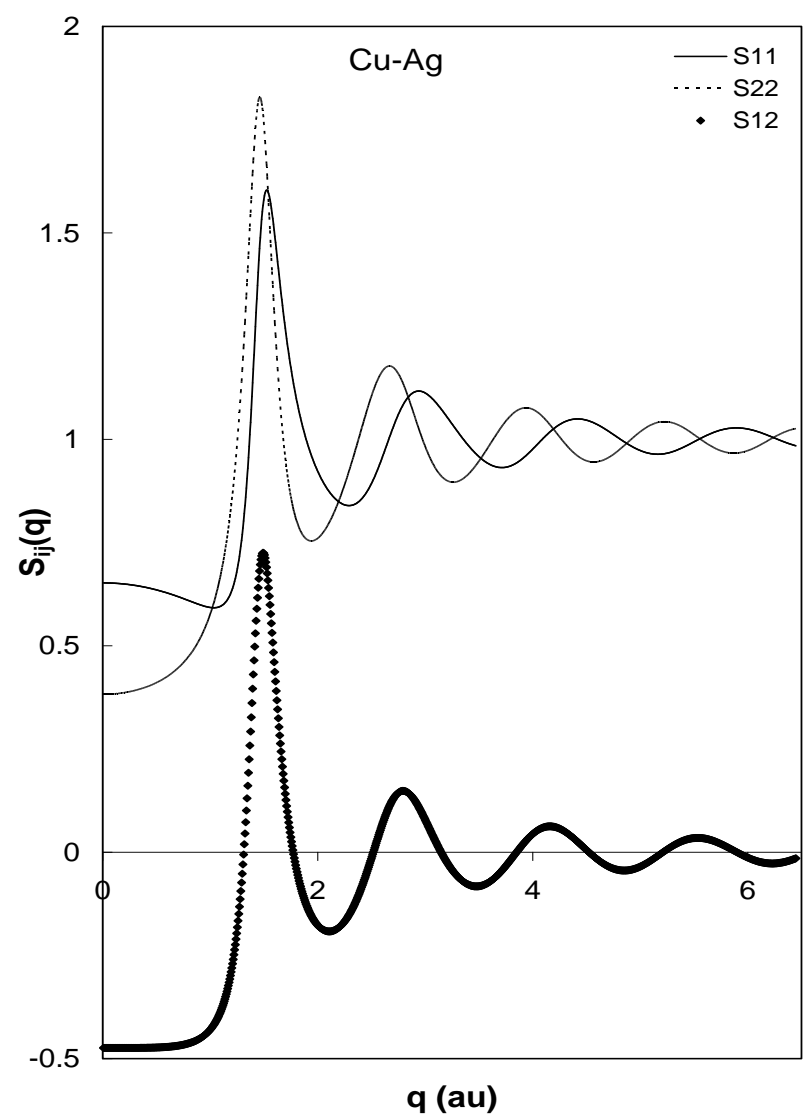

Fig. 1. Partial structure factors $\left(S_{i j}(q)\right)$ for $\mathrm{Cu}-\mathrm{Ag}$ equiatomic alloys.

The presently computed partial structure factors $\left(S_{i j}(q)\right.$ ) of equiatomic liquid noble metal alloys are shown in Fig. 1-3. From the Fig. 1-3 it is seen that, the partial structure factor of unlike atom pairs $(i-j)$ has maxima, which lower than those of two like atom papers (i-i, j-j). All the partial structure factors $\left(S_{i j}(q)\right)$ show a random mixing without a subpeak or asymmetric of the first peak. The equiatomic liquid noble metal alloys generally show endothermic reaction in the heat of mixing and its absolute value $(\Delta E)$ is small $[35,36]$. Also, one can be noted that, when we go from $\mathrm{Cu}-\mathrm{Ag} \rightarrow \mathrm{Cu}-\mathrm{Au}$ alloys, the peak positions of the partial structure factors
$\left(S_{i j}(q)\right.$ ) shifts downwards direction. The highest peak observed in the case of $\mathrm{Cu}-\mathrm{Ag}$ alloy. Also, one can observed from the Fig. 1-3 that, the effect of second metallic component shows highest peak in the structure factors of noble metals alloys.

Ashcroft and Langreth $[35,36]$ have pointed out that the core radius of the bare ion potential $W_{B}(q)$ was fitted to the experimental data at $q=2 k_{F}$, and therefore there is uncertainty in the behaviour of $W_{B}(q)$ at $q=0$. Therefore, $\alpha_{i}$ of equation (11) may be viewed as an independent parameter can be chosen to fit some further property, say, internal energy $(F)$ for pure liquid, and then this parameter may be used in the alloy calculation. Jordar et al. [8] have been pointed out that, such a calculation in the case of the $\mathrm{Ag}-\mathrm{Cu}$ alloy is possible but in view of the unavailability of volume shrinkage data, which is not necessary in the calculation.

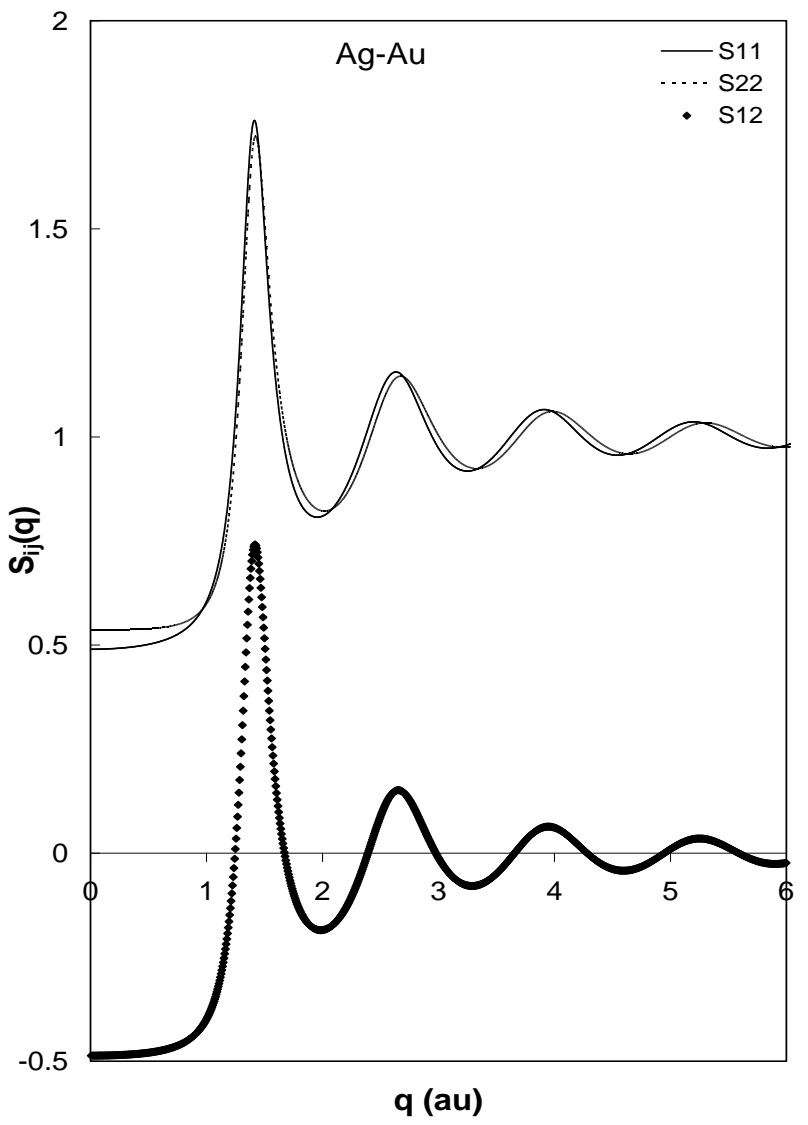

Fig. 2. Partial structure of $\left(S_{i j}(q)\right)$ for Ag-Au equiatomic alloys.

But, in the present case, we have adopted here model potential parameter $r_{C}$ from the first zero of the form factor, which gives the thermodynamic properties of liquid noble metal binaries in the range of the theoretical or experimental data [8] wherever exist. Therefore, at $q=0$, the uncertainty is totally removed in the presently used model potential. In the present computation, we have 
avoided any fitting procedure to choose the model potential parameter $r_{C}$.

Thus, in this work we have shown that a well-known local model potential with an appropriate dielectric screening functions can be applied to the equiatomic liquid noble metal alloys with reasonable accuracy. But, the validity of pseudopotential theory for liquid alloys is questionable, particularly with the evidence that this theory becomes less reliable in alloy systems where the valence difference between both metallic substances increases [8]. The PY hard sphere structure factors for these liquid noble metal alloys are found quite satisfactory. Thus this calculation shows an energy separation between non-structural and structural energies expressed as a sum of pair wise interactions between the ions. Indeed, the quantitative difference between the experimental and present structure factors which is not much very strongly reflected in the thermodynamic properties of the equiatomic liquid noble metal alloys because of the fact that these properties depend on integrals of the structure factors $[8]$.

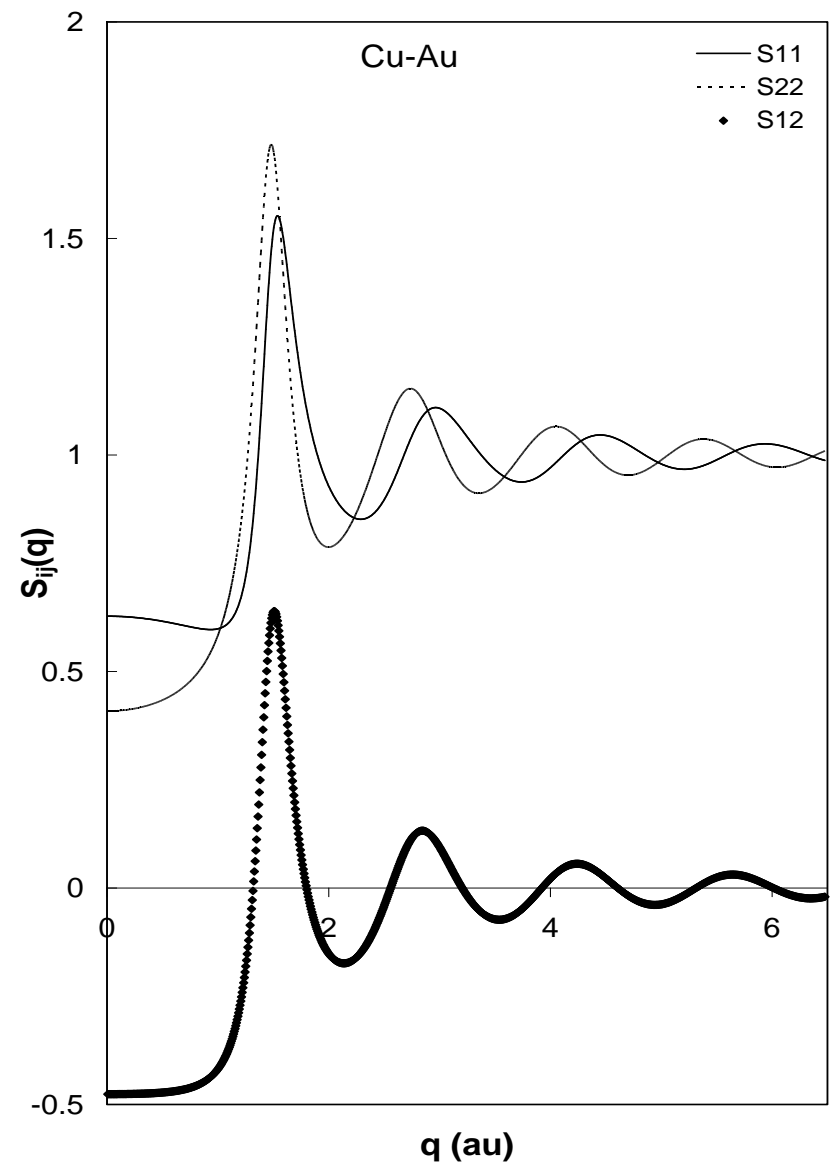

Fig. 3. Partial structure factors $\left(S_{i j}(q)\right.$ ) for $\mathrm{Cu}$-Au equiatomic alloys.

\section{Conclusion}

Finally, we concluded that, the GB technique is successfully used in the present study of the thermodynamic properties of equiatomic liquid noble metal alloys. It is found that, the properties are sensitive to the form of the model potential used, structural part of the energy, the form of the local field correction functions and volume of the mixing. The different forms of the local field correction functions have little affected on the total internal energy, Helmholtz free energy and the heat of mixing. Thus, the proper choice of the model potential along with the local field correction function is very less affected on the thermodynamic properties of binary alloys. The thermodynamics of the with five local field correction functions due to HR, TY, IU, FD and SS gives comparable results of the excess entropies and heat of mixing. This confirms the applicability of our well known model potential in explaining the thermodynamics of equiatomic liquid noble metal alloys. Such study on thermodynamic in other metals and their alloys are in progress and will communicate in near future.

\section{Reference}

1. Hafner, J.: Phys. Rev. A 1977, 16, 351. DOI: $10.1103 /$ PhysRevA.16.351

2. Singh, R. N.; Sommer, F. Rep. Prog. Phys. 1997, 60, 57. DOI: $10.1088 / 0034-4885 / 60 / 1 / 003$

3. Umar, I. H.; Young, W. H. J. Phys. F: Met. Phys. 1974, 4, 525. DOI: $10.1088 / 0305-4608 / 4 / 4 / 010$

4. Silbert, M.; Umar, I. H.; Watabe, M.; Young, W. H. J. Phys. F: Met. Phys.1975, 5, 1262.

DOI: $10.1088 / 0305-4608 / 5 / 7 / 005$

5. Stroud, D. Phys. Rev. B 1973, 7, 4405.

DOI: $\underline{10.1103 / \text { PhysRevB.7.4405 }}$

6. Umar, I. H.; Mayer, A.; Watabe, M.; Young, W. H. J. Phys. F: Met Phys. 1974, 4, 1691.

DOI: $\underline{10.1088 / 0305-4608 / 4 / 10 / 016}$

7. Stevenson, D. J. Phys. Rev. B 1975, 12, 3999 DOI: $10.1103 /$ PhysRevB.12.3999

8. Joardar, R. N.; Palchaudhuri, S.; Gopala Rao, R. V. Phys. Rev. B 1984 30, 4417.

DOI: $10.1103 /$ PhysRevB.30.4417

9. Umar, I. H.; Watabe, M.; Young, W. H. Philos. Mag. 1974, 30, 957. DOI: $10.1080 / 14786437408207249$

10. Umar, I. H.; I. Yokoyama, Young, W. H. Philos. Mag.1976, 34, 535. DOI: $10.1080 / 14786437608223792$

11. Badirkhan, Z.; Akinlade, O.; Pastore, G.; Toshi, M. P. J. Phys. Conden. Matt. 1992, 4, 6173 DOI: $10.1088 / 0953-8984 / 4 / 29 / 003$

12. Mishra, A. K.; Singh, R. N.; Sahay, B. B. Phys. Chem. Liq. 1993, 25, 153

DOI: $\underline{10.1080 / 00319109308030357}$

13. Dubinin, N. E.; Yuryev, A. A.; Vatolin, N. A. Thermochimica Acta 1998, 316, 123 . DOI: $10.1016 / \mathrm{S} 0040-6031(98) 00312-8$

14. Mishra, S. C.; Srivastava, P. L. Phys. Stat. Sol. (b) 1982, 109, 457. DOI: $10.1002 /$ pssb. 2221090203

15. Singh, R. N. J. Phys. F: Met. Phys. 1980, 10, 1411. DOI: $10.1088 / 0305-4608 / 10 / 7 / 010$

16. Vora, Aditya M. Phys. Chem. Liq. 2008, 46, 278. DOI: $10.1080 / 00319100701716536$

17. Vora, Aditya M. Inter. J. Theor. Phys., Group The. Nonlinear Opt. 2008, 13, 67.

18. Vora, Aditya M. J. Engg. Phys. Thermophys. 2009 82,779 . DOI: $10.1007 / \mathrm{s} 10891-009-0250-5$

19. Vora, Aditya M. J. Engg. Phys. Thermophys. 2010, 83, 1070. DOI: $\underline{10.1007 / \mathrm{s} 10891-010-0432-1}$

20. Vora, Aditya M. Romanian J. Phys. 2010, 55, 137.

21. Vora, Aditya M. Commun. Theor. Phys. 2010, 54, 551. DOI: $10.1088 / 0253-6102 / 54 / 3 / 33$

22. D. Chekmarev, M. Zhao, S. Rice, Structure of the liquid-vapor interface of a metal from a simple model potential: Corresponding states of the alkali metals, J. Chem. Phys. 109 (1998) 768-778. 
23. M. H. Ghatee, M. Bahadori, Inter-ionic potential function in liquid alkali metals by thermodynamic regularity approach, Chem. Phys. 335 (2007) 215-220.

24. Gajjar, P. N.; Vora, A. M.; Jani, A. R. Mod. Phys. Lett.B, 2004, 18, 573.

DOI: $10.1142 / \mathrm{S} 0217984904007104$

25. Gajjar, P. N.; Vora, A. M.; Jani, A. R. Ind. J. Phys., 2004, 78, 775.

26. Harrison, W. A. Elementary Electronic Structure; World Scientific: Singapore, 1999.

27. Taylor, R. J. Phys. F: Met. Phys. 1978, 8, 1699. DOI: $10.1088 / 0305-4608 / 8 / 8 / 011$

28. Ichimaru, S.; Utsumi, K. Phys. Rev. B 1981, 24, 3220. DOI: $10.1103 /$ PhysRevB.24.3220

29. Farid, B.; Heine, V.; Engel, G. E.; Robertson, I. J. Phys. Rev. B 1993, 48,11602 DOI: $10.1103 /$ PhysRevB.48.11602

30. Sarkar, A.; Sen, D. S.; Haldar, S.; Roy, D. Mod. Phys. Lett. B 1998, 12,639 . DOI: $10.1142 / \mathrm{S} 0217984998000755$

31. Yadawa, P. Adv. Mat. Lett. 2011, 2157 DOI: $10.5185 /$ amlett.2010.12190

32. Thakor, P. B.; Sonvane, Y.A.; Gajjar, P. N.; Jani A.R. Adv. Mat. Lett. 2011, 2303.

DOI: $10.5185 /$ amlett.indias. 200

33. Adhikari D.; Jha I. S.; Singh B. P. Adv. Mat. Lett. 2012, 3, 226. DOI: $10.5185 /$ amlett.2012.3324

34. Waseda, Y. The Structure of Non-Crystalline Materials; McGraw-Hill Int. Book Com.: New York, 1980.

35. Ashcroft, N. W.; Langreth, D. C. Phys. Rev. 1967, 159, 500. DOI: $\underline{\text { 10.1103/PhysRev. } 159.500}$

36. Ashcroft, N. W.; Langreth, D. C. Phys. Rev. 1967, 156, 685. DOI: $10.1103 /$ PhysRev.156.685

37. Moriarty, J. A. Phys. Rev. B 1970, 1, 1363. DOI: $\underline{10.1103 / P h y s R e v B .1 .1363}$

38. Heine, V.; Weaire, D. in Solid State Physics, Ehrenreich, H. et al.(Eds.); Academic Press: New York, 1970, Vol. 24.

\section{Advanced Materials Letters}

\section{Publish your article in this journal}

ADVANCED MATERIALS Letters is an international journal published quarterly. The journal is intended to provide top-quality peer-reviewed research papers in the fascinating field of materials science particularly in the area of structure, synthesis and processing, characterization, advanced-state properties, and applications of materials. All articles are indexed on various databases including $\mathrm{DOAJ}$ and are available for download for free. The manuscript management system is completely electronic and has fast and fair peer-review process. The journal includes review articles, research articles, notes, letter to editor and short communications. 